\section{Die Endokarditis ist ein Chamäleon}

\section{Unspezifisch und vielgestaltet äußern sich die Beschwerden einer Endokardi- tis. Wie lässt sich diese Erkrankung erkennen und behandeln?}

T rotz wesentlicher Fortschritte bei der Diagnostik und Therapie ist die infektiöse Endokarditis weiterhin eine Erkrankung mit ernster Prognose; die Mortalität liegt bei 10 bis $20 \%$. Die Inzidenz hat in den letzten Jahren nicht wesentlich zugenommen. Sie liegt bei $3-7 / 100.000$ Menschen. In 10 bis $30 \%$ der Fälle handelt es sich um eine Prothesenendokarditis, deren Mortalität sogar bis $\mathrm{zu} 40 \%$ beträgt. Die Symptomatik der Endokarditis ist unspezifisch und vielgestaltig. „Die Endokarditis ist ein Chamäleon“, so Prof. Klaus Matschke, Dresden. Symptome sind typischerweise Fieber, oft in Form von Schüben, Atemnot, Müdigkeit, Abnahme der körperlichen Leistungsfähigkeit, Schweißausbrüche, Herzinsuffizienz, Schlaganfall, Herzrhythmusstörungen und zentralnervöse Symptome. Im Hinblick auf das diagnostische und therapeutische
Prozedere unterscheidet man zwischen der Endokarditis bei einer nativen Klappe und der frühen (bis ein Jahr postoperativ) bzw. der späten Prothesenendokarditis. Auch wenn der Nachweis von Vegetationen ein sicheres Diagnosekriterium ist, finden sich diese nur bei $79 \%$ der Prothesenendokarditiden und 66\% der nativen Endokarditiden.

Für das Management der Endokarditis-Patienten wird in der Leitlinie ein Endokarditis-Team gefordert. „Dieses ist zwar grundsätzlich sinnvoll, es lässt sich aber leider nicht etablieren“, meint Matschke. Er kenne keine Klinik, die über eine solche Einrichtung verfüge.

Wichtig sei, dass der Patient nicht zu spät dem Herzchirurgen vorgestellt werde. Ziele der Operation sind die Vermeidung einer progressiven Herzinsuffizienz bzw. irreversiblen infektionsbedingten Zerstörung kardialer Strukturen,

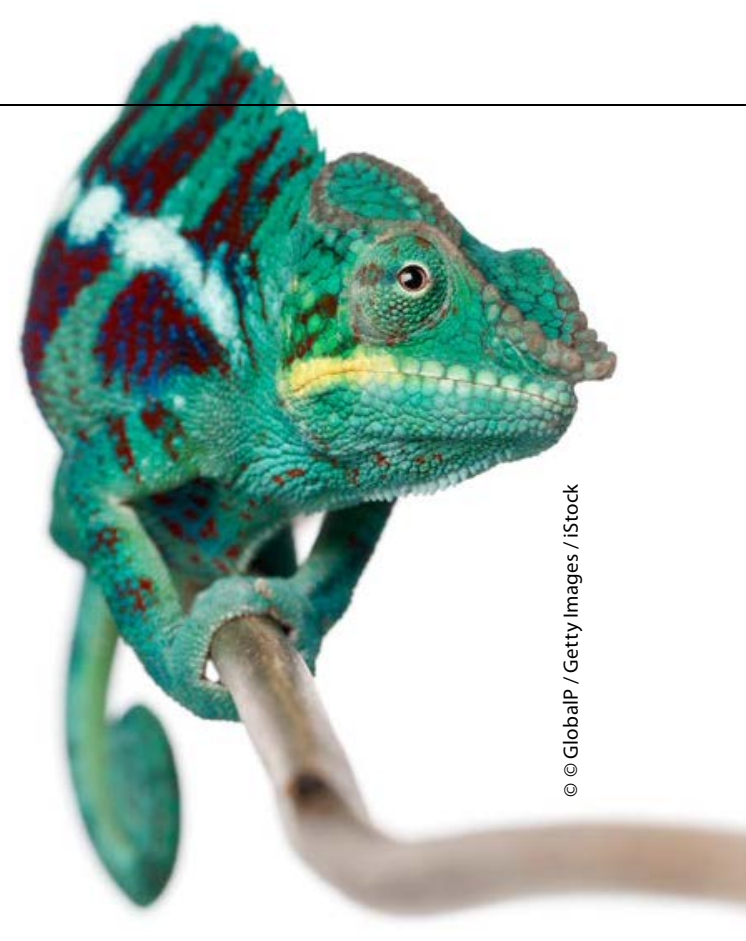

aber auch die Verhinderung von Embolien. Für letzteres gibt es kaum Daten. Eine Operationsindikation besteht, so Matschke, immer dann, wenn nach einer dreitägigen Antibiose die Blutkulturen noch positiv sind. Bei einer intrakraniellen Blutung ist die OP entgegen älterer Empfehlungen auch binnen 14 Tagen möglich. Grundsätzlich sollte die betroffene Nativ-Klappe erhalten werden, was aber nicht immer möglich ist. sti

\title{
Koronarstenose in Ruhe lassen oder nicht?
}

\begin{abstract}
Bisher gibt es keine Evidenz dafür, dass Patienten mit einer stabilen KHK und guter linksventrikulärer Funktion nach Ausschluss einer Hauptstammstenose von einer Koronarintervention prognostisch profitieren. In der ISCHEMIA-Studie wird dieser Frage jetzt erneut nachgegangen.
\end{abstract}

„ISCHEMIA ist die bisher weltweit größte Studie zur Klärung der optimalen Therapiestrategie für Patienten mit chronisch stabiler KHK und mindestens moderater Myokardischämie von mindestens $10 \%$ in einem kardialen Stress-Test", berichtete Dr. Rolf Dörr aus Dresden. Deutschland sei allerdings mit einem Randomisierungsanteil von nur $1 \%$ unterrepräsentiert. Die Patientenrekrutierung laufe noch bis zum 30.12.2017.

Eingeschlossen wurden bisher 5.655 Patienten mit stabiler KHK. Bei ihnen wird zunächst mittels CT-Angiografie eine Hauptstammstenose ausgeschlossen. Bei Patienten mit fortgeschrittener Niereninsuffizienz, bei denen ein CT nicht möglich ist, muss die Haupt- stammstenose anhand der Klinik und des Ischämieausmaßes ausgeschlossen werden. Dann erfolgt eine Ischämiediagnostik mit Belastungs-EKG, Stress-Echo, Stress-MRT oder Einzelphotonen-Emissions-CT (SPECT).

\section{Ziel der ISCHEMIA-Studie}

Bei 9,9\% der Patienten ergab sich kein Ischämienachweis und bei $9,4 \%$ fand sich eine Hauptstammstenose. „Interessanterweise fand sich bei 19,4\% der Patienten trotz Ischämienachweis keine obstruktive KHK“, so Dörr. Diese Patienten werden von der eigentlichen Studie ausgeschlossen, aber in einer Substudie weiterverfolgt. Als mögliche Ursache der Ischämie müsse bei solchen Patien- ten eine endotheliale Dysfunktion, eine "Small Vessel Disease“, eine diastolische Dysfunktion und ein Ionen-Dysfunktion diskutiert werden.

Ziel der ISCHEMIA-Studie ist es, den Stellenwert einer invasiven Therapiestrategie bei Patienten mit stabiler KHK zu evaluieren. Das Design erinnert an die COURAGE-Studie, deren Ergebnisse vor einigen Jahren Aufsehen erregten, da sie keinen Benefit für die invasive Therapie zeigten. Verglichen wird wiederum eine optimale medikamentöse Therapie plus Koronarintervention mit einer alleinigen intensiven medikamentösen Therapie. „Bisher gibt es keinen Trend zugunsten eines Arms", so Dörr. Die Ergebnisse werden für 2019 erwartet. Die Studie wird einen maßgeblichen Einfluss auf die internationalen Leitlinien und das Management von Patienten mit chronisch stabiler KHK haben.

Dr. med. Peter Stiefelhagen

22. Dresdner Symposium „Herz und Gefäße Innovationen 2016", 19.11.2016 in Dresden 